\title{
THERMAL DISPERSION WITHIN A POROUS MEDIUM NEAR A SOLID WALL
}

\author{
T. Simon, G. McFadden, ${ }^{2}$ and M. Ibrahim ${ }^{3}$ \\ ${ }^{1}$ University of Minnesota, Minneapolis, MN, USA, 55455 \\ ${ }^{2}$ Isothermal Systems Research, Liberty Lake, WA, USA, 99019 \\ ${ }^{3}$ Cleveland State University, Cleveland, OH, USA, 44115
}

\begin{abstract}
The regenerator is a key component to Stirling cycle machine efficiency. Typical regenerators are of sintered fine wires or layers of fine-wire screens. Such porous materials are contained within solid-wall casings. Thermal energy exchange between the regenerator and the casing is important to cycle performance for the matrix and casing would not have the same axial temperature profile in an actual machine. Exchange from one to the other may allow shunting of thermal energy, reducing cycle efficiency. In this paper, temperature profiles within the near-wall region of the matrix are measured and thermal energy transport, termed thermal dispersion, is inferred. The data show how the wall affects thermal transport. Transport normal to the mean flow direction is by conduction within the solid and fluid and by advective transport within the matrix. In the near-wall region, both may be interrupted from their normal in-core pattern. Solid conduction paths are broken and scales of advective transport are damped. An equation is presented which describes this change for a wire screen mesh. The near-wall layer typically acts as an insulating layer. This should be considered in design or analysis. Effective thermal conductivity within the core is uniform. In-core transverse thermal effective conductivity values are compared to direct and indirect measurements reported elsewhere and to 3D numerical simulation results, computed previously and reported elsewhere. The 3-D CFD model is composed of six cylinders in cross flow, staggered in arrangement to match the dimensions and porosity of the matrix used in the experiments. The commercial code FLUENT is used to obtain the flow and thermal fields. The thermal dispersion and effective thermal conductivities for the matrix are computed from the results.
\end{abstract}

\section{Nomenclature}

$\begin{array}{ll}\mathrm{H} & \text { Distance of temperature gradient, } \mathrm{m} \\ \overline{\bar{n}} & \text { Unit tensor } \\ \mathrm{n} & \text { Shape parameter } \\ \mathrm{r} & \text { Radial position (from the centerline), } \mathrm{m} \\ \mathrm{u} & \text { Local velocity vector, } \mathrm{m} / \mathrm{sec} \\ \mathrm{y} & \text { Position normal to the wall, } \mathrm{m} \\ \mathrm{y}_{\mathrm{m}} & \text { Dimensionless cross-stream } \\ & \text { effective conductivity } \\ \mathrm{z} & \text { Dimensionless streamwise position } \\ \theta & \text { Dimensionless temperature } \\ \phi & \text { Porosity }\end{array}$

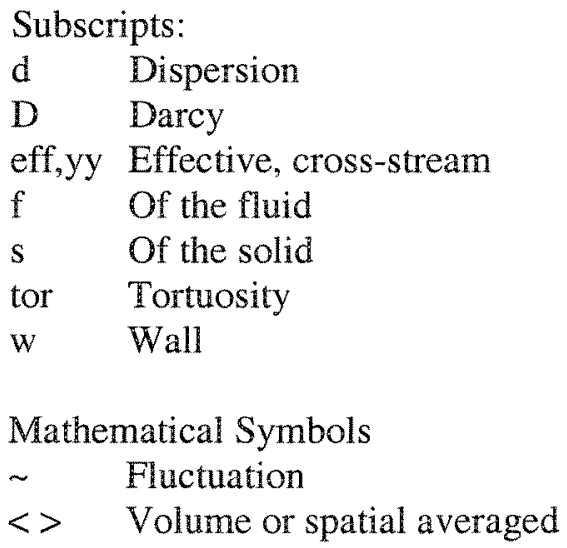

\section{Introduction}

A typical Stirling cycle regenerator geometry is a high-porosity medium made of fine wires inside of an annular solid-walled casing. During the cycle of a Stirling machine, the casing and the adjacent porous medium may not have the same axial temperature profile, which may lead to heat 
transfer between the porous medium and the casing. This heat transfer may degrade the cycle thermal efficiency below that of an ideal engine in which the matrix is isolated from the casing. Thus, it is imperative that the mechanism for heat transfer from matrix to casing is well understood.

In the case of convective heat transfer within a porous medium interrupted by an impermeable wall, one can first consider the region that is too far from the wall to be influenced by it. Within this region, there is thermal transport due to both 1) hydrodynamic mixing of the fluid within the pores, including that by eddies created as a result of flow separating from the solid elements, termed thermal dispersion, and 2) conduction through the solid and fluid phases of the porous material. Within this region, one can volume average the energy equation over the two phases (solid and fluid) of the porous medium.

The energy equation for the fluid phase is:

$$
\left(\rho c_{p}\right)_{f}\left[\frac{\partial T_{f}}{\partial t}+\nabla \cdot\left(\vec{u}_{f} T_{f}\right)\right]=\nabla \cdot\left(k_{f} \nabla T_{f}\right)
$$

while the energy equation for the solid phase is:

$$
(\rho c)_{s}\left[\frac{\partial \mathrm{T}_{\mathrm{s}}}{\partial \mathrm{t}}\right]=\nabla \cdot\left(\mathrm{k}_{\mathrm{s}} \nabla \mathrm{T}_{\mathrm{s}}\right)
$$

One now can volume average over a Representative Elementary Volume (REV) and, if one assumes local thermal equilibrium, a single governing energy equation results, as described by Whitaker (1999) and Nakayama and Kuwahara (2000).

$$
\begin{aligned}
& \frac{\partial}{\partial \mathrm{t}}\left[\phi\left(\rho c_{\mathrm{p}}\right)_{\mathrm{f}}+(1-\phi)(\rho c)_{s}\right](\mathrm{T}\rangle+\left(\rho c_{\mathrm{p}}\right)_{\mathrm{f}} \nabla \cdot \phi\langle\overrightarrow{\mathrm{u}}\rangle\langle\mathrm{T}\rangle= \\
& \nabla^{2}\left(\phi \mathrm{k}_{\mathrm{f}}+(1-\phi) \mathrm{k}_{\mathrm{s}}\right)\langle\mathrm{T}\rangle+\nabla \cdot \frac{1}{V} \int\left(\mathrm{k}_{\mathrm{f}} \tilde{\mathrm{T}}_{\mathrm{f}}-\mathrm{k}_{\mathrm{s}} \tilde{\mathrm{T}}_{\mathrm{s}}\right) \mathrm{ds}-\nabla \cdot\left(\rho c_{\mathrm{p}}\right)_{\mathrm{f}}\langle\tilde{\mathrm{T}} \tilde{\mathrm{u}}\rangle
\end{aligned}
$$

The second to the last term in equation (3), the left hand side of equation (4), represents the volumetric heat conduction through the phases of the porous medium. It is strongly dependent on the structure of the porous medium. This term accounts for the tortuous path that energy must follow as it passes through the two phases. It is condensed into another expression by using the tortuosity conductivity, $\overline{\bar{k}}_{\text {tor }}$.

$\nabla \cdot \frac{1}{\mathrm{~V}} \int\left(\mathrm{k}_{\mathrm{f}} \tilde{\mathrm{T}}_{\mathrm{f}}-\mathrm{k}_{\mathrm{s}} \tilde{\mathrm{T}}_{\mathrm{s}}\right) \mathrm{ds}=\overline{\overline{\mathrm{k}}}_{\text {tor }} \nabla\langle\mathrm{T}\rangle$

The first two terms on the right hand side of equation (3), combined with equation (4), are cast as an effective stagnant conductivity, $\overline{\bar{k}}_{0}$.

$\overline{\overline{\mathrm{k}}}_{0}=\left(\phi \mathrm{k}_{\mathrm{f}}+(1-\phi) \mathrm{k}_{\mathrm{s}}\right) \overline{\overline{\mathrm{n}}}+\overline{\overline{\mathrm{k}}}_{\text {tor }}$

The last term of equation (3) takes the thermal dispersion due to fluid advection into account. It is cast in terms of a dispersion conductivity, $\overline{\overline{k_{d}}}$. 
Equations (5) and (6) are often combined to create a total effective conductivity, $\overline{\mathrm{k}}_{\text {eff }}$. Using it results in the following equation:

$$
\frac{\partial}{\partial \mathrm{t}}\left[\phi\left(\rho c_{p}\right)_{\mathrm{f}}+(1-\phi)(\rho c)_{s}\langle\mathrm{~T}\rangle+\left(\rho c_{\mathrm{p}}\right)_{\mathrm{f}} \nabla \cdot \phi\langle\overrightarrow{\mathrm{u}}\rangle\langle\mathrm{T}\rangle=\nabla^{2} \overline{\bar{k}}_{\mathrm{eff}}\langle\mathrm{T}\rangle\right.
$$

Let us reconsider the porous medium. Within this porous medium there are solid wires and a moving fluid. Far from the wall, this fluid will exhibit transport of energy normal to the streamwise direction due to interstitial, or pore scale, mixing. However, as we are volume averaging over the fine detail, this value should remain uniform within the core of the porous medium, and will be reflected by a single value of the total effective conductivity, $\overline{\mathrm{k}}_{\text {eff }}$ within the porous medium.

Consider a region of fluid close to the impermeable boundary, or casing. When one moves radially inward from the wall through this region, one passes from a $0 \%$ porosity wall to the porosity of the core, $90 \%$ in the present study (which is typical of a Stirling cycle engine regenerator). Near the wall, the uniformity of the matrix and its properties will be disturbed. Even though the edges of the screen layers, which are stacked to form the porous matrix, are snugly against the casing wall the conduction path is broken (the matrix and the wall are not fused together). This will result in a contact resistance at the wall, not present in the core of the matrix. The fluid flow around wires in the near vicinity of the wall may also be modified. The flow resistance may be altered as a result of the geometry change and its effects on the advective transport of momentum within the near-wall porous material. This is probably predominantly due to dampening of eddies near the casing wall. We would expect this to be present at distances from the wall that are of the scale of the largest eddies within the matrix, which will be a few hydraulic diameters of the matrix. The change in eddy transport near the wall will also affect the cross-stream transport of thermal energy over the same layer. Also, the contact resistance is high compared to the resistance due to conduction within the matrix over the same distance. This is expected when the conductivity of the solid phase is high relative to the conductivity of the fluid phase, as is the case with air and stainless steel in the present experiment and helium and stainless steel in a representative Stinling engine. Due to these near-wall features, one expects the cross stream component of effective conductivity to vary radially from a value approaching the molecular conductivity of the fluid very near the wall to the sum of the effective molecular conductivity plus dispersion of the in-core matrix.

The streamwise developments of near-wall momentum and thermal fields resemble boundary layer behavior, but with a particular distribution of effective conductivity. Consider a boundary layer flow uninfluenced by gravity while assuming slug flow far from the casing (due to the effective mixing within the porous medium). As given by Burmeister (1993), the energy equation becomes:

$$
\rho\left(\frac{\partial\left(c_{v} T\right)}{\partial t}+(\vec{u} \cdot \nabla) h\right)=-p \nabla \cdot \vec{u}+\nabla \cdot(k \nabla T)+\mu \Phi
$$

If one applies that equation to a steady state, cylindrical, two-dimensional geometry with unidirectional flow and a variable cross-stream effective conductivity, $k$, a negligible pressure work term effect, $\mathrm{p} \nabla \cdot \overrightarrow{\mathrm{u}}=0$, negligibly axial conduction, no viscous dissipation, $\Phi=0$, and then volume average the result, one obtains equation (9), where all length variables are nondimensionalized on the cylinder's radius, $r_{0}$. Measurements indicate that the velocity is nearly uniform, even near the wall, in the case of this matrix and the flow conditions of the present experiment (McFadden, 2006). 
$\langle\mathrm{u}\rangle \mathrm{r}_{0}\left(\rho c_{\mathrm{p}}\right)_{\mathrm{f}} \frac{\partial\langle\theta\rangle}{\partial \mathrm{z}}=\frac{1}{\mathrm{r}} \frac{\partial}{\partial \mathrm{r}}\left(\mathrm{rk}(\mathrm{r}) \frac{\partial\langle\theta\rangle}{\partial \mathrm{r}}\right)$

where

$\theta=\frac{\langle T\rangle-T_{w}}{\left\langle T_{i}\right\rangle-T_{w}}$

and $\mathrm{T}_{\mathrm{i}}$ is the inlet temperature to the test section and $\mathrm{z}$ is the dimensionless streamwise position. In the following, $\mathrm{z}=0$ will be the location of onset of heating at the inlet to the test section.

Consider a ratio of the local cross-stream effective conductivity to the fluid conductivity.

$y_{m}=\frac{k_{e f f, y y}}{k_{f}}$

Recognizing that $y_{m}$ must vary from a value of near unity at the casing wall to some uniform value away from the wall and that equation (9) requires that $\mathrm{y}_{\mathrm{m}}$ be once differentiable, an exponential decay form is chosen. This is consistent with the exponentially decaying model proposed by Metzger et al. (2004).

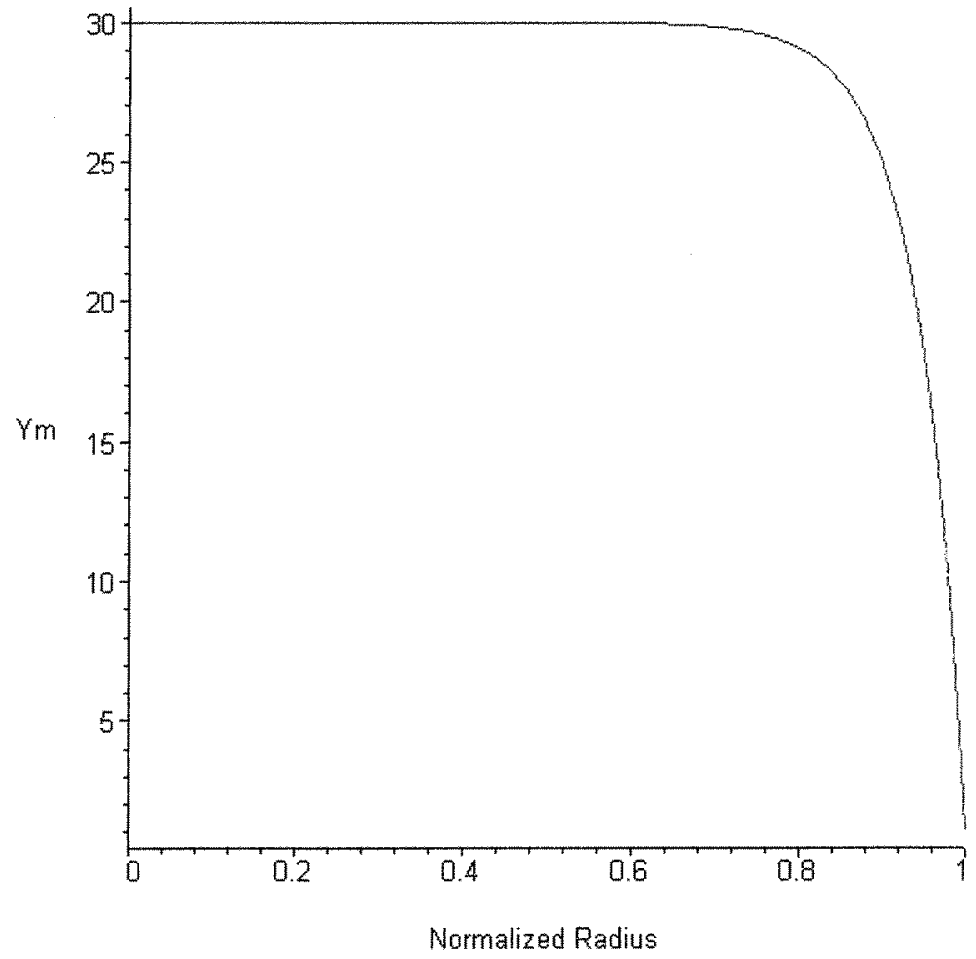

Figure 1: Example Radial Effective Conductivity Distribution 
$y_{m}=\frac{k_{\text {eff }, y y}(r)}{k_{f}}=\frac{C_{1}+C_{2} e^{-n(1-r)}}{k_{f}}$

Equations (13) and (14) are the constants which meet our boundary conditions and $\mathrm{n}$ will be chosen to fit the profile of the radial temperature distribution.

$$
\begin{aligned}
& C_{1}=\frac{e^{n} k_{e f f, y y, \infty}-k_{f}}{e^{n}-1} \\
& C_{2}=\frac{e^{n}\left(k_{e f f, y y, \infty}-k_{f}\right)}{e^{n}-1}
\end{aligned}
$$

This results in a radial relative conductivity distribution of the form shown in Figure 1. Of note is that for an $n$ value of 10 , the wall-affected region is more than 5 hydraulic diameters thick. Larger values of $\mathrm{n}$ lead to thinner wall-affected regions. It remains to determine which $\mathrm{n}$ value is suitable.

As mentioned, measurements of the exit plane velocity distribution show for this case that the average velocity is uniform across the entire matrix, even in the near-wall region. Equation (6) shows that the dispersion conductivity (a component of total effective conductivity) takes into account any thermal transport by advection, including velocity fluctuations due to eddies within the matrix. This term, when taken with equation (5), results in an effective conductivity that includes the thermophysical properties of the porous medium, the geometry of the porous medium, and the cross-stream advective transport of thermal energy.

Equation (9) is solved numerically by using Maple $8_{\circledast}$ to compute a temperature profile. Some example solutions are shown in Figure 2. This model allows for steep drops in temperature near the wall with relatively shallow curvature further into the core. For comparison, a profile with a uniform $\mathrm{k}$ is shown. The boundary conditions applied for this solution are 1) symmetry at the axial centerline, 2) uniform inflow temperature, and 3) measured wall axial temperature distribution.

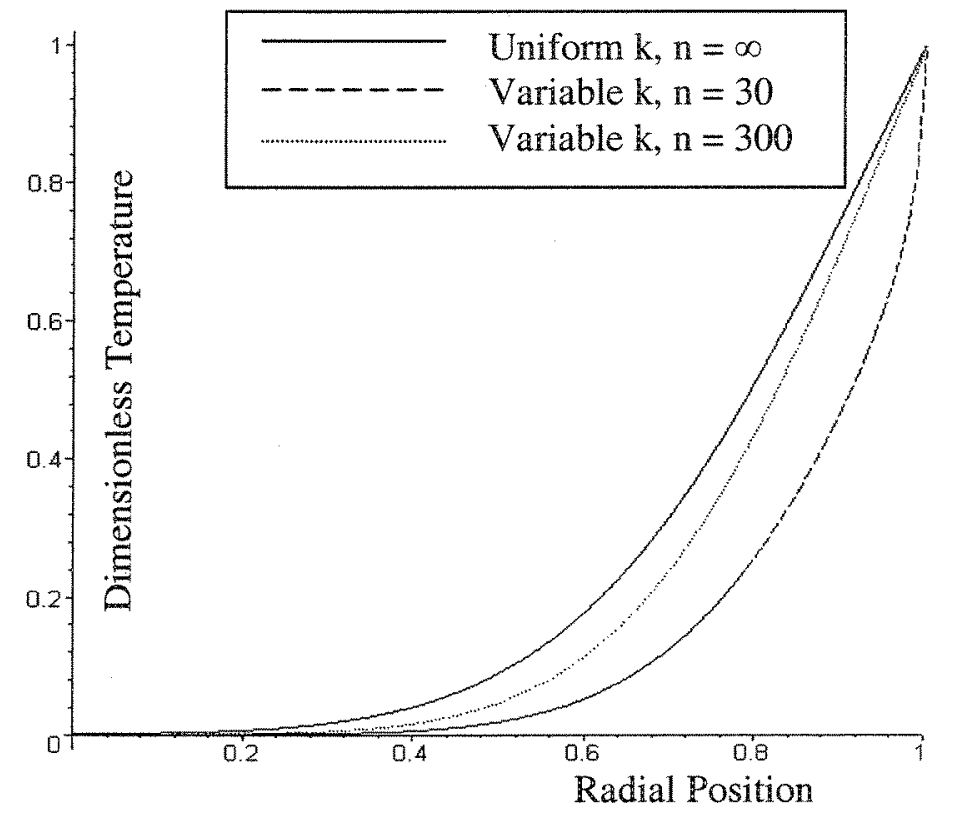

Figure 2: Temperature profiles computed with two $n$ values, and one with uniform $\mathrm{k}$. 
Previous attempts have been made by Niu et al. (2006) and Niu et al. (2005) to describe the cross stream transport terms by direct means, indirect means, and computation, all in the same matrix geometry and with the same materials and all under the same flow conditions. Niu et al. (2006) described the transport terms by using simultaneous measurements of the three components of fluctuating velocity of the flow departing the porous medium. Niu et al. (2005) indirectly determined the cross stream thermal dispersion within a porous medium using data taken within a spreading thermal wake embedded in the porous medium. Temperature measurements were taken inside the porous medium in the same manner as utilized in the present study. Velocity was unform.

\section{Measurements}

The $\mathrm{n}$ value is determined by comparison of computed temperature profiles with measured profiles in the matrix. The experiment is in a steady flow that is uniform in temperature and velocity upon entry to the matrix. The stainless steel wall of the experiment is heated. Temperature profiles are measured at various streamwise locations from the onset of heating to the matrix exit. The wall is instrumented to provide the wall thermal boundary condition. Temperature traverses are taken radially at discrete streamwise locations. The inflow temperature of the fluid is used as a reference point to normalize any inflow temperature fluctuations. Measurements indicate that one may assume a uniform velocity throughout the matrix for this case.

The temperature traverses are made using a $0.05-0.1 \mathrm{~mm}$ diameter thermocouple probe that is inserted between layers of the porous medium. This probe is inserted until it makes contact with the far wall of the test section, as verified by an electrical continuity check between the casing wall and the thermocouple bead. The last centimeter of this travel is done at a rate of about $0.1 \mathrm{~mm} / \mathrm{s} \mathrm{so}$ as to not bend or damage the thermocouple bead. Forty or more temperature readings are taken at each radial location. The traverse starts at the wall and moves toward the core initially by $0.01 \mathrm{~mm}$ steps using a traversing mechanism. Relative location within the profile is found with $0.001 \mathrm{~mm}$ precision. As the probe leaves the region near the wall, the step size is increased to reduce the quantity of data taken. The total traverse path is $100 \mathrm{~mm}$ long, which is past the centerline of the test section $(95 \mathrm{~mm})$. Figure 3 shows a diagram of the entire test section setup. The test section is a $317 \mathrm{~mm}$ long by $190 \mathrm{~mm}$ diameter stainless steel casing filled with 200 layers of $158 \times 158$ (per meter) square, welded mesh constructed of round wires of $0.81 \mathrm{~mm}$ diameter. Each successive layer is rotated $45^{\circ}$ relative to its neighbors. The test section porosity is $90 \%$. The test section wall is heated over the same region as the probe access region shown in Figure 3.

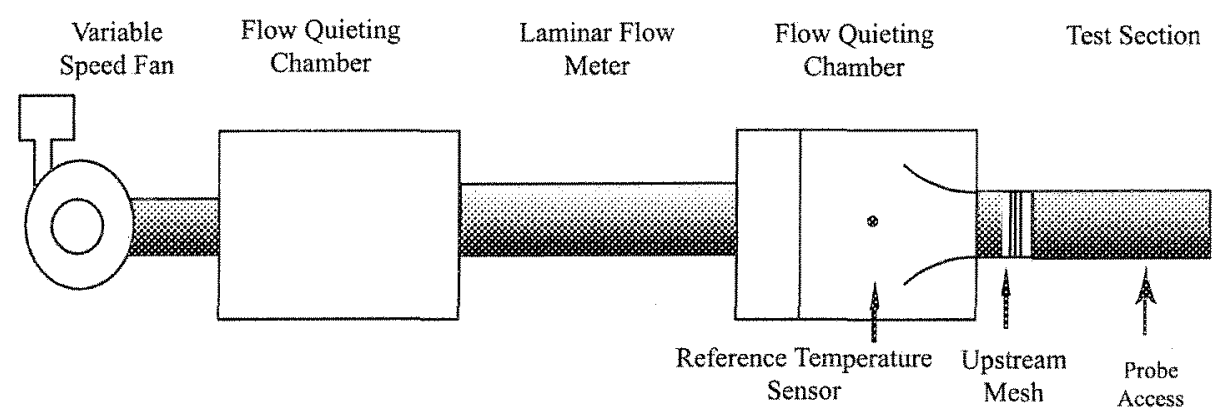

Figure 3: Test Section Setup. Matrix is a cylindrical casing filled with 220 layers of $4 \times 4$ mesh (4 wires per inch) welded screens each rotated $45^{\circ}$ from it predecessor while stacking. 


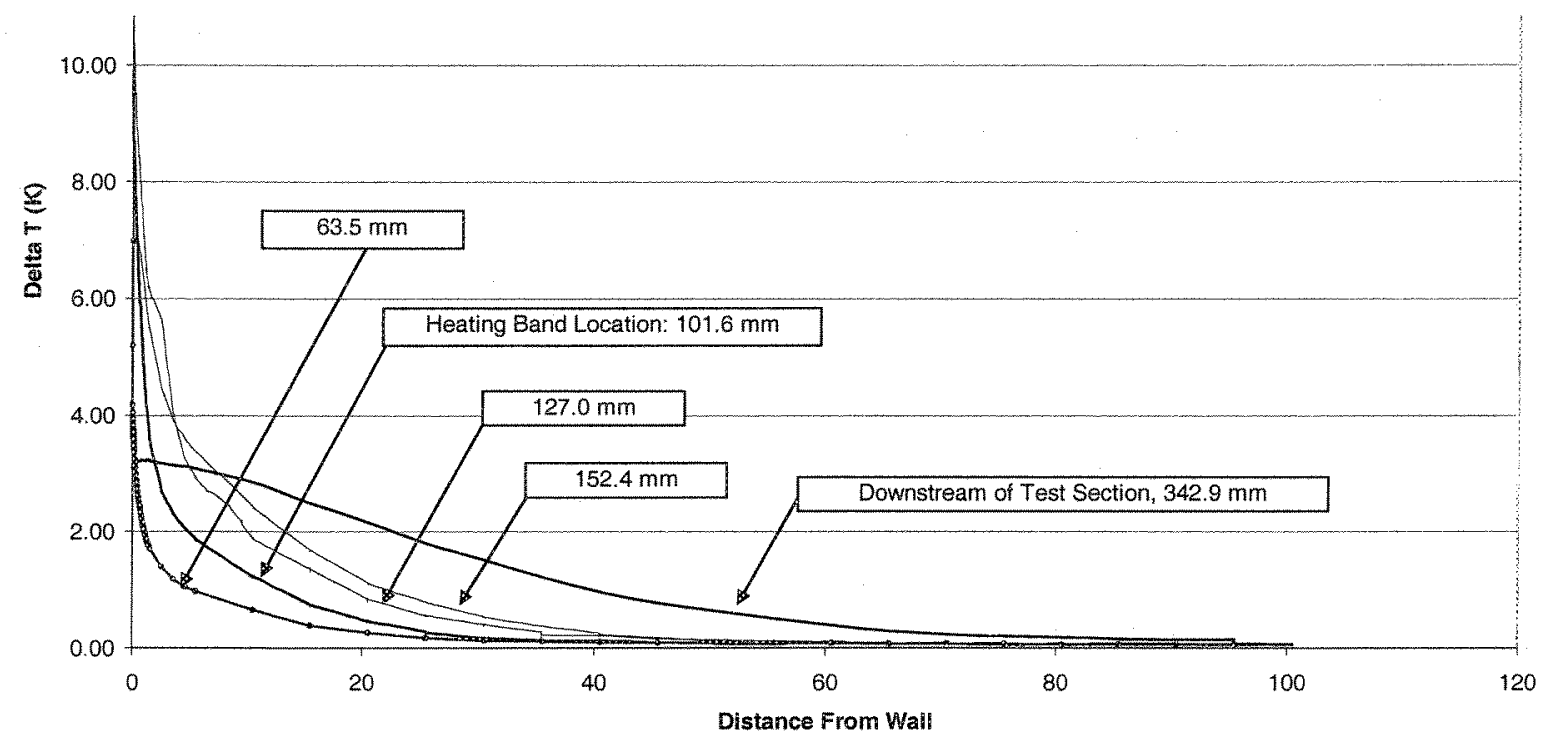

Figure 4: Experimental profiles of temperature vs radial position from the wall in mm. Temperatures are referenced to the inlet flow temperature.

\section{Experimental Results}

Measurements are taken at the axial locations referenced in Figure 4, measured from the inlet of the test section, which is also the onset of heating. The temperature difference measurement uncertainty is $0.05^{\circ} \mathrm{C}$ and the position uncertainty is $<0.1 \mathrm{~mm}$. These are based on a $95 \%$ confidence interval. One can notice the steep drop in temperature at the wall followed by a shallowing curve as one moves toward the core, as speculated in the previous section.

Figure 5 shows that with the proper choice of $n$, an excellent agreement between the analytic solution developed previously and the measured thermal profiles can be obtained.

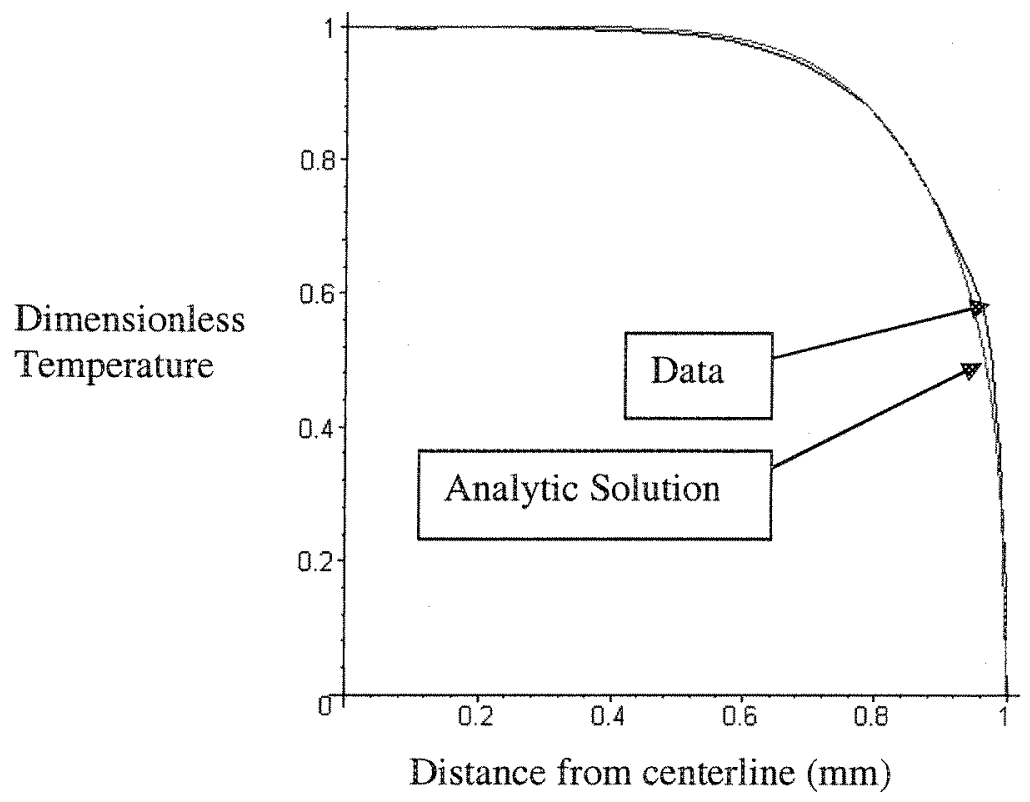

Figure 5: Dimensionless temperature versus radial distance from the centerline. A representative comparison between computed and measured temperature profiles. 


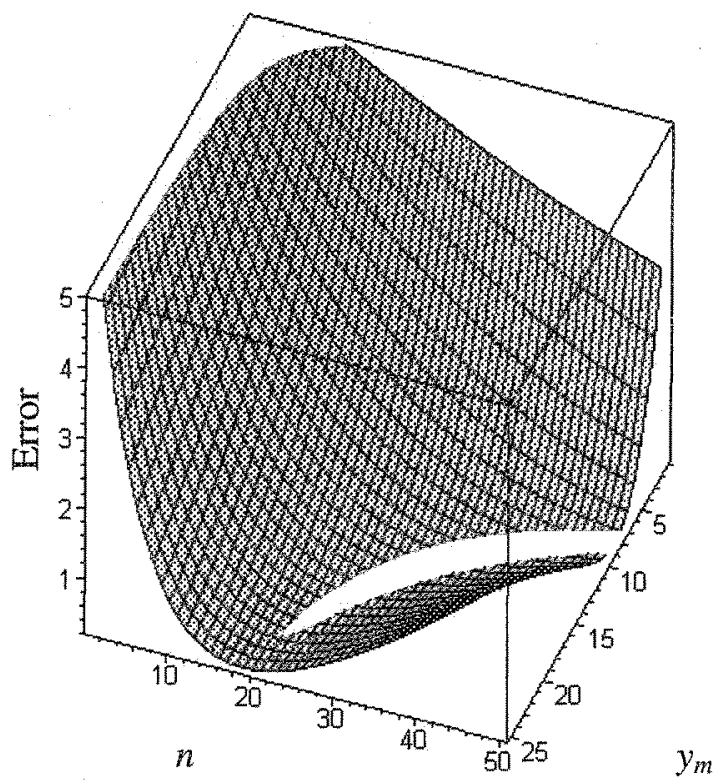

Figure 6: Example of an error plot

One must consider the accuracy with which the solution is obtained. There are two variables that will be solved, the core dispersion and the curvature controlling variable, $\mathrm{n}$. This is done by computing a large range of possible values for core $y_{m}$ and $n$ and comparing the experimental profiles to the analytical profiles. The best fit establishes $y_{m}$ and $n$. From this, a plot such as Figure 6 is generated. The white area shown in Figure 6 is a region of minimum error in $y_{m}, n$ coordinates. The error is the sum of the squares of the deviations between the predicted results from the analytic solution and the points in the experimental thermal profile. For instance, take one experimental profile. A matrix of possible solutions is computed from the analysis over a range of $y_{m}$ and $n$ values. Then a comparison is made by subtracting solution values from experimental values for each point of the profile. A difference is computed over a range of $y_{m}$ and $n$ values. The difference at each data point is squared and summed with the others for that single combination of $y_{m}$ and $n$ to get a net error value for each set of $y_{m}$ and $n$. This is plotted, as shown, for all combinations of $y_{m}$ and $\mathrm{n}$. Notice that as $\mathrm{n}$ becomes large, meaning that the thickness of the low effective conductivity region is very small, the value of $y_{m}$ decreases. Also, as the insulating layer at the wall thickens (decreasing $n$ ) the relative importance of $y_{m}$ increases.

The results from this technique are listed in Table 1. In-core values of conductivity are plotted in Figure 7 (three points at $\mathrm{Pe}=102$ ). For comparison, an independent, indirect measurement of effective conductivity in the core of the same matrix was previously made with a different technique and was reported by Niu et al. (2005) and Simon and McFadden (2006). This technique is a steady state version of one documented by Metzger et al. (2004). These in-core, indirectlymeasured values are also given in Figure 7. The eddy component of the parameter $y_{\mathrm{m}}$ (essentially, the slope of the effective in-core conductivity vs. Pe line) was measured previously by a direct means and was reported by Niu et al. (2006). These results are also shown in Figure 7. As noted, this gives only the slope of the line for the in-core effective conductivity. A stagnant conductivity (intercept) of 30 was assumed for the purpose of plotting the line based on these direct measurements. 


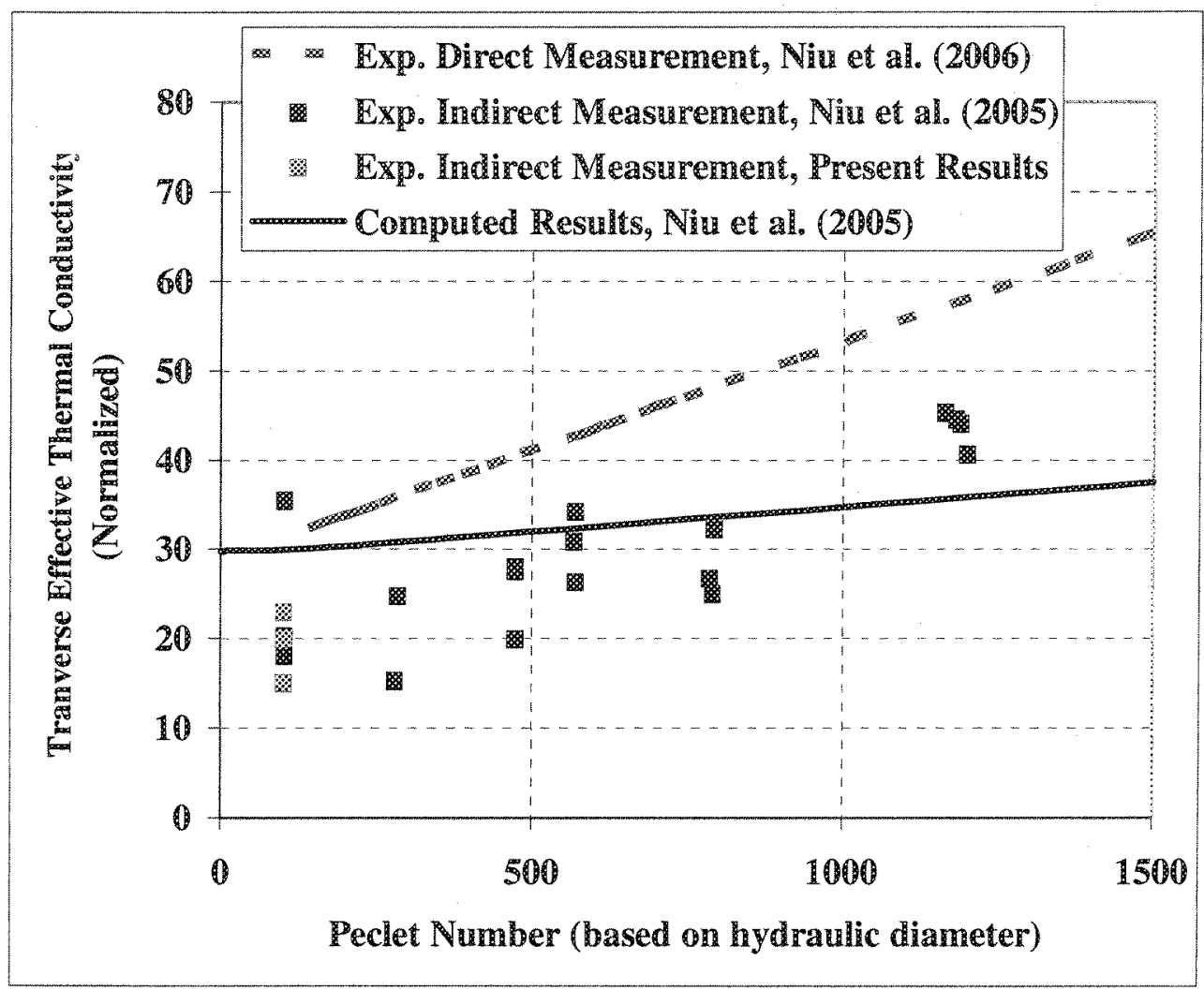

Figure 7: In-core transverse effective thermal conductivity (summation of transverse stagnant conductivity and transverse thermal dispersion) versus Peclet number (for $\mathrm{k}_{s} / \mathrm{k}_{\mathrm{f}}=673$ "stainless steel" and porosity $=0.9$ ). Compared are the present data, another set of indirect measurements taken by an independent technique (Niu et al. 2005), direct measurement results by Niu et al. 2006, and computed values from Niu et al. 2005.

Table 1: Shape Factor and Cross Stream Conductivity for $\mathrm{Pe}=102$.

\begin{tabular}{|c|c|c|}
\hline Location & $y_{m}$ Core & $n$ \\
\hline $152.4 \mathrm{~mm}$ & 23 & 24 \\
\hline $127 \mathrm{~mm}$ & 20 & 25 \\
\hline $101.6 \mathrm{~mm}$ & 15 & 25 \\
\hline
\end{tabular}

\section{Numerical Studies}

Plotted in Figure 7 are conductivity values computed with CFD. These values come from Niu et al. 2005 , but are briefly discussed below. Two different approaches are typically used in numerical modeling of heat transfer in porous media: 1) a high-resolution analysis of the matrix microstructure, and 2) a continuum and homogeneous analysis based on the spatially-averaged Representative Elementary Volume (REV). In this analysis, the first was done by considering a 3D periodic wire arrangement in which each wire is welded with three others to form a square layer. These layers are staggered with respect to adjacent layers as they are stacked, as shown in Figure 8. When a periodic boundary condition is applied, the array represents a repeating section of the matrix. This simulation is a replication of the $4 \times 4$ mesh used in the experiments discussed above, except that it does not have the $45^{\circ}$ rotation of each successive layer. The FLUENT 6.2.16 package was applied to obtain the numerical solution of the flow and temperature fields inside the matrix. This CFD model is believed to be the first 3-D model to represent such a geometry, earlier attempts 
were confined to 2-D arrays or arrays with rectangular wires (Nakayama and Kuwahara, 2000). The analysis starts with a periodic velocity condition and a linear inflow temperature profile. After solving for the flow and thermal fields, outflow velocity and temperature results are taken back into the inflow boundary condition and the solver is reloaded, after verifying proper mass flow rate. This is repeated until a periodic thermal field is achieved based on equation (15). It usually takes no more than 10 iterations to obtain approximately periodic thermal boundary conditions,

$\theta=\frac{T_{\text {inlet }}-T_{\text {wall }}}{T_{\text {bulk, inlet, }}-T_{\text {wall }}} \approx \frac{T_{\text {outlet }}-T_{\text {wall }}}{T_{\text {bulk, outlet, }}-T_{\text {wall }}}$

where $T_{b u l k}=\frac{\int T|\rho \vec{A} \cdot d \vec{A}|}{\int_{A}|\rho \vec{u} \cdot d \vec{A}|}, \quad T_{\text {inlet }}$ and $T_{\text {outlet }}$ are averaged values.

After solving for the temperature distribution inside the 3D cell, the $y$-direction effective thermal conductivity due to thermal dispersion and stagnant conductivity are calculated. The flow-normal component of the dispersion, $\overline{\mathrm{k}}_{\mathrm{eff}, \mathrm{yy}}$, is computed with the following equation:

$\overline{\mathrm{k}}_{\text {eff }, y y}=\frac{-\frac{\left(\rho c_{p}\right)_{f}}{V}}{\frac{\Delta \mathrm{T}}{\mathrm{H}}} \iiint_{\mathrm{V}}(\mathrm{T}-\langle\mathrm{T}\rangle)\left(\mathrm{v}-\langle\mathrm{v}\rangle_{\mathrm{f}}\right) \mathrm{dV}$

Where $\mathrm{v}$ is the velocity vector, $\overrightarrow{\mathrm{u}}$, projected in the $\mathrm{y}$ direction and $\mathrm{H}$ represents the distance over which the temperature gradient is imposed.

A parametric study was carried out to investigate the influence of Peclet number on the thermal dispersion conductivity in the $3 \mathrm{D}$ cell. Parameter ranges were: $\operatorname{Pr}=0.707,0<\operatorname{Pe}<1500, \phi=0.9$,

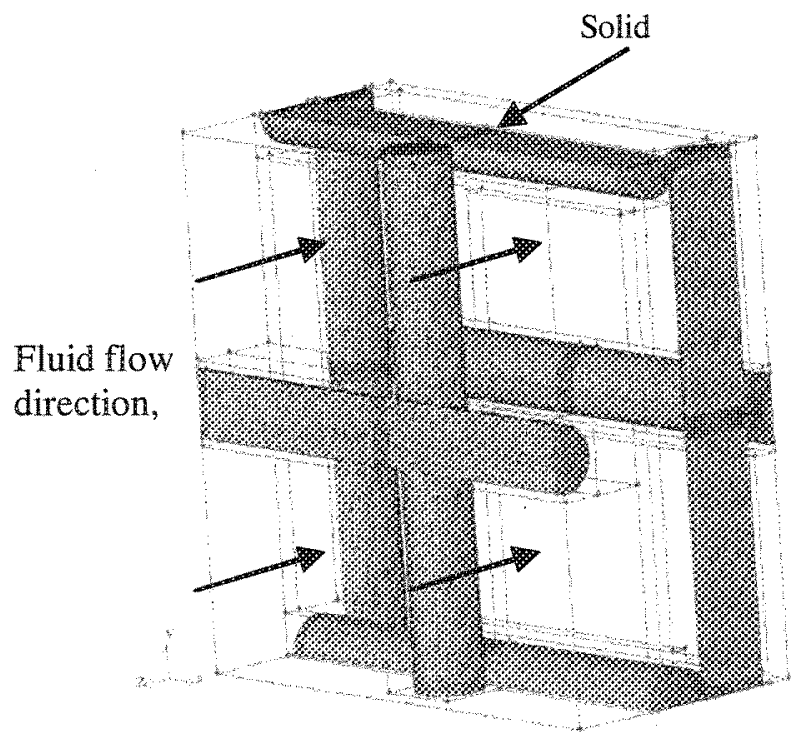

Figure 8: 3D Cell representation for CFD 
and, $\mathrm{k}_{\mathrm{s}} / \mathrm{k}_{\mathrm{f}}=673$ and $\left(\rho c_{\mathrm{p}}\right)_{\mathrm{s}} /\left(\rho c_{\mathrm{p}}\right)_{\mathrm{f}}=3273$ (stainless steel). One case, without flow, is used to compute the stagnant thermal conductivity. In successive cases, the mass flow rate is varied to compute thermal dispersion versus Peclet number. The effective lateral thermal conductivity plotted in Figure 7 is calculated by adding thermal dispersion and stagnant thermal conductivity. There is general agreement with data from the indirect and direct experimental measurements discussed above. The computed thermal dispersion component (the slope on Figure 7) from the calculation is $\mathrm{k}_{\text {eff }, y y} / \mathrm{k}_{\mathrm{f}}=0.0047 \mathrm{Pe}$. The Peclet number length scale is the matrix hydraulic diameter.

\section{Conclusion}

Table 1 shows that if there were no effects of the wall, the cross stream thermal transport would be essentially uniform. Our data show this to be generally equivalent to values found by another indirect measurement method, direct measurement, and computation. The present results also show there are lower values of cross-stream thermal transport near the wall. This is expected since the conduction path at the wall is not continuous. A model is given for the radial variation of crossstream thermal transport near an impermeable wall. Its development assumes uniform flow velocity throughout the matrix. This assumption is supported for this case by our measurements of matrix exit velocity distributions. This variability of near-wall transport will significantly affect the heat transfer between the porous medium of a regenerator and the casing wall within which it resides. Such variability must be considered in an analysis of a Stirling cycle regenerator.

\section{References}

Burmeister, L., 1993, Convective Heat Transfer, John Wiley \& Sons, Inc., 1993.

McFadden, G., 2006. "Forced Thermal Dispersion within a Representative Stirling Engine Regenerator," M.S. Thesis, Mechanical Engineering Department, University of Minnesota, 2005.

Metzger, T., Didierjean, S., and Maillet, D., "Optimal Experimental Estimation of Thermal Dispersion Coefficients in Porous Media," International Journal of Heat and Mass Transfer, Vol. 47, No. 14-16, 2004, pp. 3341-3353.

Nakayama, A. and Kuwahara, F., 2000, "Numerical Modeling of Convective Heat Transfer in Porous Media Using Microscopic Structures," Handbook of Porous Media, edited by Vafai, K., Marcel Dekker Inc., New York, 2000.

Niu, Y. McFadden, G., Simon, T., Ibrahim, M., and Rong, W., 2005, "Measurements and Computation of Thermal Dispersion in a Porous Medium," $3^{\text {rd }}$ International Energy Conversion Engineering Conference, AIAA-2005-37923, Aug. 15-18, 2005, San Francisco, CA.

Niu, Y., Simon, T., Gedeon, D., and Ibrahim, M., 2006, "Direct Measurements of Eddy Transport and Thermal Dispersion in High-Porosity Matrix," AIAA J. of Thermophysics and Heat Transfer, Vol. 20, No. 1, January-March, 2006.

Simon, T. and McFadden, G., 2006. "Forced Thermal Dispersion within a Representative Stirling Engine Regenerator," to become a NASA-CR.

Whitaker, S., 1999, The Method of Volume Averaging. Kluwer Academic Publishers, Netherlands. 